\title{
Relación entre las enfermedades más frecuentes y el rendimiento académico de los estudiantes de la Universidad Nacional José María Arguedas
}

\section{Relation between the most frequentdeseases and the academic performance of the students of the national university José María Arguedas}

\author{
Sandra Salazar Palomino
}

Universidad Nacional José María Arguedas.

\begin{abstract}
Resumen
La presente Investigación presenta resultados de como las diversas enfermedades que afectan a los estudiantes de la Universidad Nacional José maría Arguedas de Andahuaylas pueden influenciar en su rendimiento académico. Las enfermedades más frecuentes que padecen los estudiantes son: Admigdalitis, Anemia, Bronquitis, Cefalea, Contusión, Delgadez, Faringitis, Faringoadmigdalitis, Gastritis, Geca, I.T.U. , Resfriado, Sinusitis, de las cuales, el mayor porcentaje correspondió a la Faringitis con $24.2 \%$, Gastritis con $18.7 \%$. En relación a los promedios o notas de rendimiento promedio para el año 2009, según la muestra analizada estas oscilaron entre 10 y 15 , siendo el de mayor frecuencia la nota de 12. Al relacionar las notas promedio con las enfermedades, se observó que las notas promedio más bajas corresponden a los estudiantes que presentaron cuadros de bronquitis, con notas promedio de 9.14 , observándose además que los promedios para cada una de las enfermedades no alcanzan a llegar a la nota de 13 , lo cual es un indicador del bajo rendimiento. Según el análisis de regresión lineal múltiple utilizando como variables independientes las enfermedades más frecuentes (2 casos), los resultados nos infieren que si existe correlación pero en un nivel bajo, y según al análisis de pearson, la bronquitis ( $99 \%$ de confianza) y la contusión (95\% de confianza) alcanzaron una correlación inversa altamente significativa con los promedios o notas obtenido. Siendo la bronquitis la enfermedad que determina los promedios más bajos durante el semestre académico, lo cual nos indicaría que estudiante con bronquitis es candidato a no ser promovido.

Descriptores: enfermedades y rendimiento académico
\end{abstract}

\begin{abstract}
The present research shows results as how the many sickness affect to the students of José María Arguedas, University of Andahuaylas. How it can influence in their academic performance.

The more frequent sickness that the students suffer are: tonsolitis, anemia, bronchitis, cefalea, bruise, skiness, pharyngeal, pharyngeal-tonsilitis, gastritis. G.E.C.A, I.T.U, cold, sinuosity, about all of them, which has the highest percent belonged to the pharyngeal, with $24,2 \%$, the gastritis has $18,7 \%$. According their rating average to the year 2009. According the analyzed sample, these are oscillated between 10 and 15 .

Being with the most frequency the reting 12. Matching the rating average with the sickness, we can observ that the lowest rate average belongs to the students who had bronchitis, with rating average of 9.14, watching besides that the rating average with each sickness, it doesn't get to the rating of 13 , which it is an indicator at low academic performance.

According the multiple analysis of line regression, using as an independent variable the prost frequent sickness (2 cases), the results we deduce that if it exists correlation but in a low level, according the pear son analysis, the bronchitis (99\% truth) and the bruise (95\% truth), they get an inverse correlation highly meaningful with the rating average gotten. Being the bronchitis as the sickness which bring about the lowest rating average during the academic semester, which the student who has bronchitis is one of them to not be advanced.
\end{abstract}

Key words: Sickness and academic performance. 


\section{Introducción}

En consonancia con los propósitos de la investigación, es necesario conceptuar el rendimiento académico. Para ello se requiere previamente considerar dos aspectos básicos del rendimiento: el proceso de aprendizaje y la evaluación de dicho aprendizaje. Sobre la evaluación académica hay una variedad de postulados que pueden agruparse en dos categorías: aquellos dirigidos a la consecución de un valor numérico (u otro) y aquellos encaminados a propiciar la comprensión (insight) en términos de utilizar también la evaluación como parte del aprendizaje. La primera categoría, se expresa en los calificativos escolares. Las calificaciones son las notas o expresiones cuantitativas o cualitativas con las que se valora o mide el nivel del rendimiento académico en los alumnos. Las calificaciones escolares son el resultado de los exámenes o de la evaluación continua a que se ven sometidos los estudiantes. Medir o evaluar los rendimientos escolares es una tarea compleja que exige del docente obrar con la máxima objetividad y precisión [1].

En el sistema educativo peruano, en especial en las universidades la mayor parte de las calificaciones se basan en el sistema vigesimal, es decir de 0 a20 [2]. Sistema en el cual el puntaje obtenido se traduce a la categorización del logro de aprendizaje, el cual puede variar desde aprendizaje bien logrado hasta aprendizaje deficiente.

Como ya sabemos la educación escolarizada es un hecho intencionado y, en términos de calidad de la educación, todo proceso educativo busca permanentemente mejorar el aprovechamiento del alumno. En este sentido, la variable dependiente clásica en la educación escolarizada es el rendimiento o aprovechamiento escolar [3]. El rendimiento en sí y el rendimiento académico, también denominado rendimiento escolar, son definidos por la Enciclopedia de Pedagogía / Psicología de la siguiente manera: "Del latín reddere (restituir, pagar) el rendimiento es una relación entre lo obtenido y el esfuerzo empleado para obtenerlo. Es un nivel de éxito en la escuela, en el trabajo, etc. al hablar de rendimiento en la escuela, nos referimos al aspecto dinámico de la institución escolar. Al estudiar científicamente el rendimiento, es básica la consideración de los factores que intervienen en él. Por lo menos en lo que a la instrucción se refiere, existe una teoría que considera que el rendimiento escolar se debe predominantemente a la inteligencia; sin embargo, lo cierto es que ni siquiera en el aspecto intelectual del rendimiento, la inteligencia es el único factor, al analizarse el rendimiento escolar, deben valorarse los siguientes factores como la familia, la sociedad, el ambiente escolar y la salud" [4].

Resumiendo, el rendimiento académico es un indicador del nivel de aprendizaje alcanzado por el alumno, por ello, el sistema educativo brinda tanta importancia a dicho indicador. En tal sentido, el rendimiento académico se convierte en una "tabla imaginaria de medida" para el aprendizaje logrado en el aula, que constituye el objetivo central de la educación. Sin embargo, en el rendimiento académico, intervienen muchas otras variables externas al sujeto, como la calidad del maestro, el ambiente de clase, la familia, el programa educativo, etc., y variables psicológicas o internas, como la actitud hacia la asignatura, la inteligencia, la personalidad, el autoconcepto del alumno, la motivación, etc.

La Universidad está interesada en acoger en sus aulas a los mejores alumnos, por lo que establece un proceso de selección riguroso, a fin de identificarlos e insertarlos en sus filas. Todas las universidades del país han establecido un proceso de admisión, que permita seleccionar a aquellos que poseen las cualidades necesarias para un aprendizaje exitoso en la universidad, con la consiguiente, titulación y desempeño profesional capaz, honesto y responsable. Sin embargo a pesar escoger a los "mejores", se ha visto que un gran número de alumnos admitidos en las universidades no han podido responder a las exigencias que les hubieran conducido a logros satisfactorios, teniendo muchos casos de deserción y repitencias.

La formación profesional forma parte del interés social en todo país, por varias razones, pues se necesita un número suficiente de profesionales competentes que ofrezcan sus servicios basados en altas normas éticas, que sean justos y honestos, sin embargo en el Perú la deserción y repitencia en la universidad indican con claridad que el proceso de rendimiento académico estaría asociado a diversos motivos. Muchos docentes notan aparentemente una marcada disminución año tras año en el rendimiento de los estudiantes admitidos, sin lograr explicar los factores de su aparente bajo rendimiento académico. La otra variable a investigada es la referida a las enfermedades, por lo tanto utilizaremos para este trabajo la definición que señala que: Enfermedad, es toda alteración de la salud cuyo diagnóstico y confirmación sea efectuada por un médico. Una enfermedad es cualquier trastorno anormal del cuerpo o la mente que provoca malestar y alteración de las funciones normales. Las enfermedades son entidades creadas por el hombre, en su afán de clasificar el conocimiento, en la que se agrupan una 
serie de componentes que la definen y que se debe distinguir de los síndromes y los síntomas. En ocasiones, algunos síndromes o síntomas se denominan erróneamente como enfermedades, especialmente en el caso de la psiquiatría. Debido a que cada ser humano es único y que el componente emocional y socio ambiental se añade al componente físico, siempre se ha dicho que no existen las enfermedades sino los enfermos, por lo que cada persona tiene una forma de enfermar diferente a otra, a pesar de padecer la misma enfermedad.

Considerando que a la fecha existen pocas investigaciones puntuales sobre la relación entre enfermedades más frecuentes y el rendimiento académico en estudiante universitarios se tomará en cuenta como referentes los estudios e investigaciones relacionados con enfermedades y rendimiento a nivel escolar y superior como los realizados en la Universidad de Salamanca (España), donde se investigó ¿Por Qué Disminuye El Rendimiento Académico? señalándose que las principales causas del bajo rendimiento es 'la dificultad intrínseca de algunas materias', 'el excesivo número de asignaturas que los alumnos tienen que cursar cada año', la 'extensión desproporcionada de los programas' y el 'elevado número de exámenes y trabajos' [5].

En el Perú, se cuenta con la investigación sobre la "Relación entre el rendimiento académico, la ansiedad ante los exámenes, los rasgos de personalidad, el autoconcepto y la asertividad en estudiantes del primer año de psicología de la Universidad Nacional Mayor de San Marcos" donde se indica que la necesidad de obtener un adecuado rendimiento académico, puede convertirse en un factor estresante para los estudiantes [6]. Otro tema investigado trató sobre el rendimiento académico de los alumnos de la Facultad de educación de misma universidad y su percepción de la calidad académica de los docentes, donde los resultados indican que, básicamente, no existe correlaciones significativas entre ambas variables, salvo algunas que se puedan considerar como bajas [7].

Nuestra experiencia docente en la Universidad Nacional José María Arguedas, ubicada en el Departamento de Apurímac, nos evidencia que los jóvenes estudiantes acuden frecuentemente al servicio médico donde se registran las enfermedades que padecen, pero también se evidencia registros de bajo rendimiento académico, hecho que me permite plantear la interrogante ¿Qué relación existe entre el rendimiento académico y las enfermedades más frecuentes en los estudiantes de la Universidad Nacional José María Arguedas?. Investigación que permitirá descartar o aceptar la
Hipótesis que propongo que si existe una relación directa entre esta dos variables.

\section{Objetivo General}

Determinar la relación entre el rendimiento académico y las enfermedades más frecuentes en los estudiantes de la Universidad Nacional José María Arguedas - 2009.

\section{Objetivos Específicos}

Identificar cuáles son las enfermedades más frecuentes que padecen los estudiantes.

Identificar el rendimiento académico de los estudiantes.

Identificar cual es la relación entre el rendimiento académico y las enfermedades más frecuentes en los estudiantes.

\section{Metodología}

\section{Recojo de la Información}

Respecto a la información sobre la realidad problemática se ha recurrido a documentación de tipo secundario: revistas, textos, tesis, Internet.

Respecto a la recolección de datos, después de plantear los objetivos, se han determinado las variables en estudio (las enfermedades) que han sido tomadas en base a su frecuencia.

Tipo de muestreo: en el estudio se hizo un Muestreo Estratificado al Azar, solo para los estudiantes que poseían alguna enfermedad.

Procesamiento de la Información.

En el procesamiento de la información se utilizaron los programas:

1. Microsoft Excel 2003.

2. SPSS14

Análisis de la Información.

La muestra fue extraída según el Muestreo Estratificado al Azar con la siguiente formula:

$$
n_{0}=\frac{Z^{2} p^{*} q}{d^{2}} \longrightarrow n=\frac{n_{0}}{1+\frac{n_{0}}{N}}
$$

Donde:

$p=$ es la proporción de estudiantes que no padecen una enfermedad en la Universidad Nacional José maría Arguedas.

$q=$ es la proporción de estudiantes que padecen una enfermedad en la Universidad Nacional José María Arquedas. 


\section{Regresión Múltiple.}

Se utilizó regresión múltiple cuando estudiamos la posible relación entre varias variables independientes (predictoras o explicativas) y otra variable dependiente (criterio, explicada, respuesta).

\section{Aplicaciones de la Regresión Múltiple}

Es cierto que la regresión múltiple se utiliza para le predicción de respuestas a partir de variables explicativas.

Requisitos y limitaciones de la regresión múltiple Hay ciertos requerimientos necesarios para poder utilizar la técnica de regresión múltiple:

\section{Linealidad}

Se supone que la variable respuesta depende linealmente de las variables explicativas. Si la respuesta no aparenta ser lineal, debemos introducir en el modelo componentes no lineales (como incluir transformaciones no lineales de las variables independientes del modelo). Otro tipo de respuesta no lineal es la interacción. Para ello se ha de incluir en el modelo términos de interacción, que equivalen a introducir nuevas variables explicativas que en realidad son el producto de dos o mas de las independientes.

\section{Normalidad}

La validez del modelo requiere que los mismos se distribuyan de modo normal y con la misma dispersión para cada combinación de valores de las variables independientes.

\section{Número de variables independientes}

Podemos estar tentados en incluir al modelo cualquier cosa que tengamos en una base de datos, con la esperanza de que cuantas más variables incluyamos, más posibilidades hay de que nuestras hipótesis o ideas sean las correctas. Una regla que se suele recomendar es la de incluir al menos 20 observaciones por cada variable independiente que estimemos a priori interesantes en el modelo. Números inferiores nos llevaran posiblemente a no poder obtener conclusiones y errores de tipo II.

\section{Colinealidad}

Si dos valores independientes están estrechamente relacionadas y ambas son incluidas en un modelo, muy posiblemente ninguna de las dos sea considerada significativa1.

Variables numéricas e indicadoras (Dummy)

Un modelo de regresión lineal tiene el aspecto:

$Y=\beta_{0}+\beta_{1} * D_{1}+\ldots . .+\beta_{n} * D_{n}+E$

Donde:
Y: es la variable dependiente

$\mathrm{Di}$ : representa a las variables independientes Dummy.

Los coeficientes son calculados por el programa estadístico, de modo que se minimicen los residuos. Está claro que para ajustar el modelo, la variable respuesta debe ser numérica. Sin embargo, aunque pueda parecer extraño no tienen por que serlo las variables explicativas. Aunque requiere un artificio, podemos utilizar predictores categóricos mediante la introducción de variables indicadoras (también denominadas mudas o Dummy).

\section{Características de las Variables Explicativas}

Para mejor comprensión se elaboró un cuadro, donde se incluyó las variables a analizar, las mismas que fueron codificadas. En base a esta codificación podemos decir que nuestro modelo es de la siguiente forma:

$$
\begin{aligned}
Y= & \beta_{0}+\beta_{1} D_{1}+\beta_{2} D_{2}+\beta_{3} D_{3}+\beta_{4} D_{4}+\beta_{5} D_{5}+\beta_{6} D_{6}+\beta_{7} D_{7} \\
& +\beta_{8} D_{8}+\beta_{9} D_{9}+\beta_{10} D_{10}+\beta_{11} D_{11}+\beta_{12} D_{12}+\beta_{13} D_{13}+E
\end{aligned}
$$

\section{Resultados}

\section{Enfermedades más frecuentes en los estudiantes del año académico 2009.}

Los estudiantes tomados como muestra, según los registros de la Oficina de Bienestar Universitario (OBU) de la UNAJMA, presentan como enfermedades más frecuentes a la Admigdalitis, Anemia, Bronquitis, Cefalea,Contusión , Delgadez, Faringitis, Faringoadmigdalitis, Gastritis, Geca, I.T.U. , Resfriado, Sinusitis, de las cuales, el mayor porcentaje correspondió a la Faringitis con $24.2 \%$, Gastritis con $18.7 \%$, y la menos frecuente fue la Sinusitis con $2.0 \%$. ( Fig. 1.).

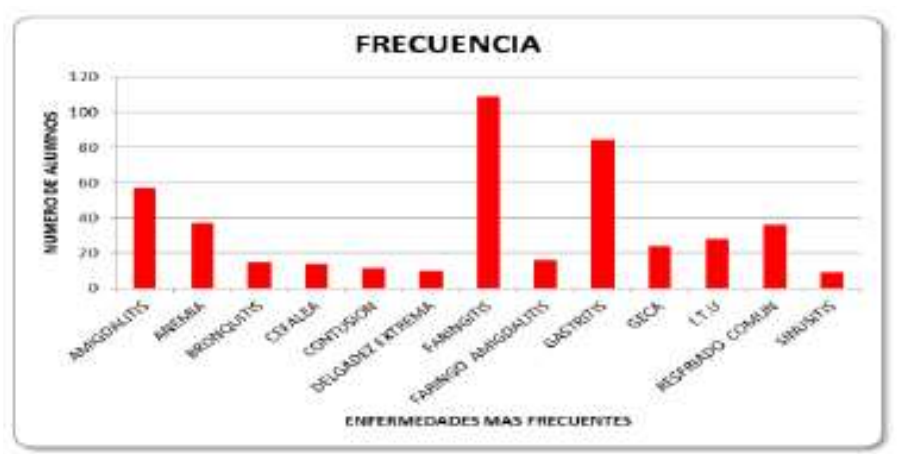

Fig. 1: Relación entre el número de alumnos versus enfermedades más frecuentes. 
Promedio de notas o rendimiento académico de los estudiantes del año académico 2009.

Los promedios o notas de rendimiento promedio para el año 2009, según la muestra analizada indican que estos oscilaron entre 10 y 15, siendo el de mayor frecuencia la nota de 12 (Fig.2).
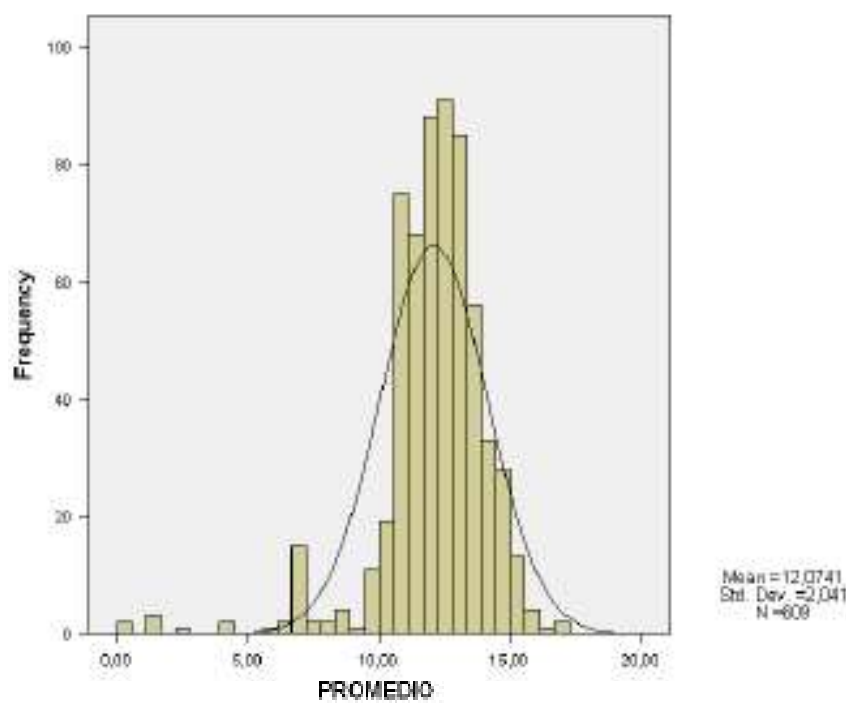

Fig. 2: Frecuencia de promedio de notas

Notas promedio versus enfermedades más frecuentes

La Fig. 3, nos muestra que las notas promedio más bajas corresponden a los estudiantes que presentaron cuadros de Bronquitis, con notas promedio de 9.14, observándose además que los promedios para cada una de las enfermedades no alcanzan a llegar a la nota de 13 , lo cual es un indicador del bajo rendimiento .

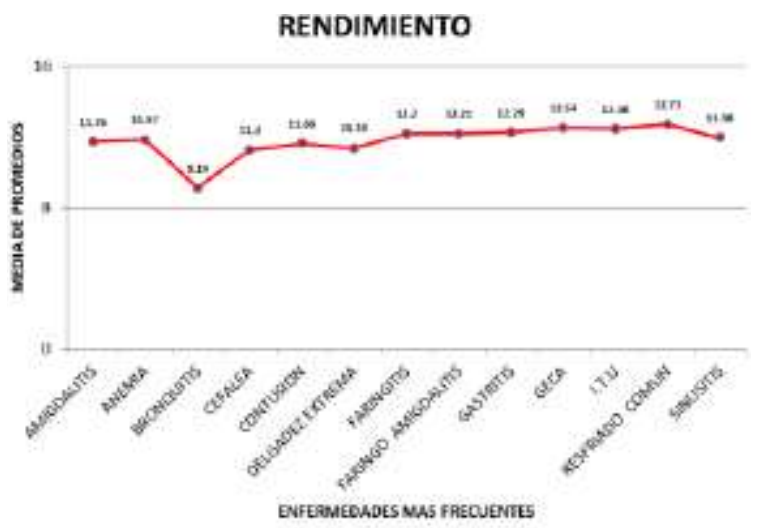

Fig. 3: Notas promedio versus enfermedades más frecuentes
Relación de enfermedades más frecuentes con el rendimiento académico

\section{Prueba de normalidad.}

Según la prueba de normalidad de K-S, nos dice que los datos se alejan ligeramente de la curva norma con un $95 \%$ de nivel de confianza, por lo tanto rechazamos la hipótesis de que los datos se distribuyen en forma normal, es decir los datos no se ajustan a una distribución normal de media 12.07 y D.S. 2.04, porque el ZK-S es mayor que la diferencia absoluta.

Cuadro 1: Prueba de normalidad One-Sample Kolmogorov-Smirnov Test

\begin{tabular}{|l|c|r|r|r|}
\hline Model & R & R Square & $\begin{array}{c}\text { Adjuste } \\
\text { d R } \\
\text { Square }\end{array}$ & $\begin{array}{c}\text { Std. Error } \\
\text { of the } \\
\text { Estimate }\end{array}$ \\
\hline 1 & $.198(a)$ & .039 & .018 & 2.02231 \\
\hline
\end{tabular}

a Predictors: (Constant), Sinusitis, delgadez, cefalea, bronquitis, faringoadmidalitis, GECA, I.T.U, contusión, resfriado, anemia, agmidalitis, gastritis, farinqitis.

Estos resultados nos infieren que si existe correlación pero en un nivel bajo (posiblemente por el número de observaciones y falta de aleatoriedad en las notas)

Cuadro 2: Variables Entered/Removed(b)

\begin{tabular}{|l|l|l|l|}
\hline Mode & \multicolumn{1}{|l|}{$\begin{array}{c}\text { Variabl } \\
\text { es } \\
\text { Entered }\end{array}$} & $\begin{array}{c}\text { Remov } \\
\text { ed }\end{array}$ & Method \\
\hline 1 & $\begin{array}{l}\text { SINUSITIS, } \\
\text { DELGADEZ, }\end{array}$ & & \\
& $\begin{array}{l}\text { CAFALEA, } \\
\text { BRONQUITIS, }\end{array}$ & & \\
& FARIGOADM, & & \\
& GECA, I.T.U, & & \\
& CONTUSION, & & Enter \\
& RESFRIADO, \\
& ANEMIA, \\
& AGMIDALITIS, \\
& GASTRITIS, \\
& FARINGITIS(a) & & \\
\hline
\end{tabular}

a All requested variables entered.

b Dependent Variable: PROMEDIO 
Cuadro 3: Resúmen del modelo (Model Summary)

\begin{tabular}{|l|r|r|r|r|}
\hline Model & R & R Square & $\begin{array}{c}\text { Adjuste } \\
\text { d R } \\
\text { Square }\end{array}$ & $\begin{array}{c}\text { Std. Error } \\
\text { of the } \\
\text { Estimate }\end{array}$ \\
\hline 1 & $.198(\mathrm{a})$ & .039 & .018 & 2.02231 \\
\hline
\end{tabular}

a Predictors: (Constant), Sinusitis, delgadez, cefalea, bronquitis, faringoadmidalitis, GECA, I.T.U, contusión, resfriado, anemia, agmidalitis, gastritis, faringitis.

\section{Análisis de varianza ANOVA(b)}

Cuadro 4: Análisis de varianza

\begin{tabular}{|l|l|r|r|r|r|r|}
\hline $\begin{array}{l}\text { Mo } \\
\text { del }\end{array}$ & & $\begin{array}{c}\text { Sum of } \\
\text { Squares }\end{array}$ & df & $\begin{array}{l}\text { Mean } \\
\text { Squar } \\
\text { e }\end{array}$ & F & Sig. \\
\hline 1 & $\begin{array}{l}\text { Regre } \\
\text { ssion }\end{array}$ & 99.325 & 13 & 7.640 & 1.86 & $.031(\mathrm{a})$ \\
& $\begin{array}{l}\text { Resid } \\
\text { ual } \\
\text { Total }\end{array}$ & $\begin{array}{l}2433.399 \\
2532.724\end{array}$ & 595 & 4.090 & & \\
608 & & & \\
\hline
\end{tabular}

a Predictors: (Constant), SINUSITIS, DELGADEZ, CAFALEA, BRONQUITIS, FARIGOADM, GECA, I.T.U, CONTUSION, RESFRIADO, ANEMIA, AGMIDALITIS, GASTRITIS, FARINGITIS

b Dependent Variable: PROMEDIO

Estos resultados muestran el nivel de significancia de las enfermedades en el rendimiento académico, observándose $P>$ que 0,005 , lo que nos indica que no hay diferencia significativa entre las variables cuando se relacionan con el promedio.

\section{Correlación Bivariada de Pearson}

De acuerdo al análisis de Pearson, la bronquitis (99\% de confianza) y la contusión $(95 \%$ de confianza) alcanzaron una correlación inversa altamente significativa con los promedios o notas obtenido. Siendo la bronquitis la enfermedad que determina los promedios más bajos durante el semestre académico, lo cual nos indicaría que estudiante con bronquitis es candidato a no ser promovido (Cuadro No. 5).

Aquí se muestran los resultados de la parte experimental, se analiza y discute los mismos frente a otros autores (nacionales o internacionales). Si el trabajo es teórico se analiza y discute los resultados del modelo o la simulación empleada [5].

\section{Discusión}

Según las investigaciones disponibles cuando se habla de rendimiento académico (éxito-retrasodeserción) en la Universidad, las explicaciones giran prioritariamente en torno a variables de tipo cognitivas. Los diagnósticos del pobre desempeño académico apuntan a problemas de insuficiencia cognitiva, mala formación en la enseñanza media para acceder a estudios superiores -niveles de conocimientos inadecuados a las exigencias universitarias; desarrollo insuficiente de aptitudes especiales acordes al tipo de carrera elegida, falta de estrategias y métodos de estudio o técnicas de trabajo intelectual, etc. También son tenidas en cuenta ciertas variables "extracognitvas" que interactúan con las cognitivas. Dentro de estas, el énfasis está puesto en los factores macrosociales (nivel cultural y académico de los padres, trabajo, etc.) que repercuten en el rendimiento académico. Se trata pues, de estudios que correlacionan variables de corte sociológico (más que psicosociales) y rendimiento. Dichos aspectos cognitivos y extracognitivos inherentes al estudiante (y condicionantes del rendimiento académico), abarcan diversos factores. Estos factores, en los mencionados estudios que atienden a una perspectiva macrosocial, pueden resumirse en: Factores personales. Factores familiares. Factores académicos. Los primeros están constituidos por aspectos descriptivos, estructurales de los sujetos: edad, sexo, estado civil, número de hijos, lugar de nacimiento, entorno de convivencia. Los resultados principales que se hallaron respecto a la influencia de dichas variables personales sobre el rendimiento académico son los siguientes: existe en general una asociación altamente significativa entre la edad y el rendimiento académico. Cuanto más joven es el ingreso, mejor es el rendimiento [9]. Por su parte, el sexo [10] y el entorno de convivencia (si vive solo o con su familia) no influyen significativamente en el éxito académico [11,12]. Si bien el rendimiento mejora en aquellos que viven con su familia; y aunque sin grandes diferencias, las mujeres abandonan más que los hombres [13,12]. Respecto al estado civil y al número de hijos los estudios muestran un elevado rendimiento de los casados sin hijos, incluso superior a los solteros. Sin embargo, la baja incidencia de alumnos casados en comparación con los solteros, impide arribar a diferencias estadísticamente significativas [12]. Los factores "familiares" aluden a los aspectos socioeconómicos, y también se consideran variables estructurales: ocupación y categoría ocupacional de los padres, escolaridad de estos, eventual trabajo del estudiante. Tampoco existiría asociación entre la variable "trabajo" (si el estudiante trabaja o no) y el rendimiento académico [14]. Con todo, refiriéndonos específicamente a las "horas de trabajo", paradójicamente, los alumnos que más horas trabajan, son los que mejor rendimiento tienen, en contra de lo que cabría suponer, ya que los que trabajan poco o no lo hacen, tendrían más tiempo para estudiar [12]. 
Según estudios realizados por la Universidad Nacional Federico Villareal, en el Perú, el bajo rendimiento, la deserción y repitencia en la Universidad indican con claridad que, en muchos casos, la base escolar ha sido débil. En otras circunstancias, el proceso de admisión no ha permitido detectar a quienes realmente servían para seguir estudios universitarios. En este sentido, un número significativo de estudiantes no ha sabido responder a las exigencias que lo hubiera conducido a logros satisfactorios en la Universidad y posterior desempeño en bien de la sociedad. Se ha sugerido factores que podrían estar asociados al bajo rendimiento, como la insuficiente vocación y capacidad para los estudios superiores; y esto es porque algunos estudiantes ingresan a la Universidad en busca de un "cartón", vale decir el título profesional. En la parte económica es conocido que algunas veces condicionan los estudios. Es pues de suma importancia considerar que los estudiantes empiezan sus carreras con un nivel de motivación muy alto y, con el transcurso del tiempo, esta motivación disminuye, traduciéndose en un bajo rendimiento académico e inclusive en abandono de los estudios, además de la existencia de otros factores de riesgo, no considerados en este estudio, como por ejemplo la personalidad, inseguridad en la carrera, falta de hábitos de estudio e inclusive por consumo de bebidas alcohólicas que en determinadas circunstancias podrían ocasionar problemas académicos y así una eventual deserción $[15,16]$.

Sin embargo, se considera que para tener un buen rendimiento académico y físico, los estudiantes universitarios necesitan consumir alimentos nutritivos ricos en vitaminas, minerales y proteínas además de evitar el desarrollo, a largo plazo, de enfermedades como desnutrición, obesidad, estreñimiento, bronquitis, entre otras. Así como problemas de visión y dolores de cabeza. Las diversas enfermedades que pueden pasar desapercibidas en el niño o adolescente y repercutir en su rendimiento académico pueden agruparse en: Enfermedades orgánicas que repercuten en el aprendizaje. Enfermedades psicológicas o psiquiátricas que afectan al estado de ánimo. Trastornos específicos del aprendizaje. El primer grupo, tal vez el más simple de resolver, se explica fácilmente exponiendo un ejemplo: el alumno que no progresa adecuadamente en la escuela, instituto y/o universidad es porque presenta algún defecto de visión. En este caso la solución suele ser sencilla una vez corregido el supuesto problema visual. Generalmente se trata de estudiantes en los que a los problemas de aprendizaje se suman otros, generados por una o varias enfermedades de carácter físico u orgánico; siendo este grupo de interés para nuestra investigación. Las enfermedades psiquiátricas son algo más complejas. En este grupo se englobarían patologías que, aunque afortunadamente son poco frecuentes, conviene tenerlas presentes, como los cuadros de depresión o ansiedad y otros procesos psiquiátricos más graves como los trastornos de conducta, los trastornos generalizados del desarrollo-espectro autista y los trastornos de la personalidad [17]. Dentro de estas enfermedades podríamos englobar, como un grupo aparte, a los estudiantes que se hallan en una situación socio-familiar adversa o aquellos con problemas de relación con sus compañeros en su centro educativo (el término aplicado recientemente es el de bullying), con dificultades para el diagnóstico del problema y de una enorme complejidad en el manejo terapéutico [18].

En este marco, son pocos los trabajos que tratan de incorporar la variable enfermedad, como otro de los elementos que ampliaría la explicación del bajo rendimiento de los estudiantes universitarios, por ejemplo un estudio realizado por la Universidad Nacional Autónoma de México [19], aseguró que jóvenes entre 17 y 21 años de edad que padecen el llamado síndrome metabólico disminuyen su rendimiento académico y obtienen bajas calificaciones. "Se trata de jóvenes que no sabían de su problema y por ello se deben impulsar campañas para realizar diagnósticos oportunos";

Los resultados de la presente investigación, muestran que los estudiantes de la Universidad Nacional José María Arguedas, tomados como muestra , presentaron como enfermedades más frecuentes a la Admigdalitis, Anemia, Bronquitis, Cefalea, Contusión , Delgadez(desnutrición), Faringitis, Faringoadmigdalitis, Gastritis, Geca, I.T.U. , Resfriado, Sinusitis, de las cuales, el mayor porcentaje correspondió a la Faringitis con $24.2 \%$, Gastritis con $18.7 \%$, y la menos frecuente fue la Sinusitis con $2.0 \%$. . Este resultado se asemeja a lo encontrado en otras investigaciones que indican que los estudiantes universitarios como cualquier otro ser humano se encuentra expuesto a adquirir "desajustes" en su salud, las mismas que están en la mayoría de los casos asociados a las condiciones del hábitat, la geografía del lugar y la climatología en particular . Debido a que cada ser humano es único y que el componente emocional y socio ambiental se añade al componente físico, siempre se ha dicho que no existen las enfermedades sino los enfermos, por lo que cada persona tiene una forma de enfermar diferente a otra, a pesar de padecer la misma enfermedad [20]. 
$\mathrm{Si}$ observamos los resultados del rendimiento académico, nos muestra que las notas promedio más bajas corresponden a los estudiantes que estuvieron enfermos de Bronquitis, con notas promedio de 9.14, observándose además que los promedios para cada una de las enfermedades no alcanzan a llegar a la nota de 13 , lo cual es un indicador del bajo rendimiento en general. Al correlacionar las variables de estudio, se comprueba mediante la prueba de Pearson que la bronquitis a un nivel de confianza del $99 \%$ y la contusión al $95 \%$ de confianza, alcanzaron una correlación inversa altamente significativa con los promedios o notas obtenidos. Siendo la bronquitis la enfermedad que determina los promedios más bajos durante el semestre académico, lo cual nos indicaría que estudiante con bronquitis es candidato a no ser promovido. La explicación porque los estudiantes que presentan el cuadro clínico de Bronquitis tienen las notas promedio más bajas, estaría asociado al tipo de enfermedad, la misma que es una inflamación de las vías aéreas bajas. Sucede cuando los bronquios, situados entre los pulmones, se inflaman a causa de una infección o por alguna otra causa, siendo sus síntomas : Tos con mucosidad, a veces sanguinolenta. Si el moco de la bronquitis es verde amarillento y va acompañada de fiebre, lo más probable es que haya infección bacteriana. Además los enfermos con esta dificultad respiratoria, presentan malestar general. En la bronquitis crónica, también pueden presentarse los siguientes síntomas: Inflamación de tobillos, pies y piernas, coloración azulada de los labios provocado por los bajos niveles de oxígeno en la sangre [21]. Además se conoce que otros factores pueden empeorar la bronquitis: La contaminación atmosférica, las alergias, ciertas ocupaciones (como la extracción de carbón, la fabricación de textiles o la manipulación de granos) [22].

Como se puede inferir entonces, estudiante con bronquitis, definitivamente esta disminuido en sus capacidad de atención por las molestias de la enfermedad y esta se complica con la no asistencia a las clases, por cuanto en la mayoría de las veces debe guardar cama. En términos generales podemos decir que los resultados obtenidos en esta investigación, son comparables con la investigación realizada con estudiantes ingresantes de la Universidad Nacional de Trujillo en el año 2004, donde se reportó que las enfermedades más frecuentes fueron las visuales (ambliopía y astigmatismo); asma crónico, moderado y leve; desnutrición aguda, leve; migraña y dolor tensional, y que al aplicar pruebas estadísticas, encontraron que los estudiantes que presentaron problemas visuales (ambliopía, estrabismo), asma moderado, desnutrición aguda, migraña, disminuyeron su rendimiento académico, sin embargo ese rendimiento fue aprobatorio, pero, los estudiantes con asma crónico tuvieron un rendimiento desaprobatorio, mientras que los estudiantes con asma leve, incrementaron su rendimiento académico aprobatoriamente [23].

En resumen de lo mencionado por diversos investigadores, podemos decir que el rendimiento académico es un indicador del nivel de aprendizaje alcanzado por el alumno. Sin embargo, en el rendimiento académico, intervienen muchas otras variables externas al sujeto, como la calidad del maestro, el ambiente de clase, la familia, el programa educativo, etc., y variables psicológicas o internas, como la actitud hacia la asignatura, la inteligencia, la personalidad, el autoconcepto del alumno, la motivación, etc. pero estamos de acuerdo en el sentido que al analizarse el rendimiento escolar, deben valorarse los siguientes factores como la familia, la sociedad, el ambiente escolar y la salud" [4].

\section{Conclusiones}

Las enfermedades más frecuentes que padecen los estudiantes de la Universidad Nacional José María Arguedas, fueron: Admigdalitis, Anemia, Bronquitis, Cefalea, Contusión, Delgadez, Faringitis, Faringoadmigdalitis, Gastritis, Geca, I.T.U. , Resfriado, Sinusitis, de las cuales, el mayor porcentaje correspondió a la Faringitis con 24.2 \%, Gastritis con $18.7 \%$.

El rendimiento académico, mostró que las notas promedio más bajas correspondieron a los estudiantes que estuvieron enfermos de Bronquitis, con notas promedio de 9.14 , observándose además que los promedios para cada una de las enfermedades no alcanzaron a llegar a la nota de 13 , lo cual es un indicador del bajo rendimiento en general.

Se puede inferir, que los estudiantes con bronquitis, de acuerdo al análisis de regresión determina los promedios más bajos durante el semestre académico, lo cual nos indicaría que estudiante con bronquitis es candidato a no ser promovido, atribuible a los efectos de la enfermedad que al complicarse ocasiona la no asistencia a las clases, por cuanto en la mayoría de las veces debe guardar cama. 


\section{Agradecimientos}

A mis padres, gracias a su apoyo constante y su amor me motivan a seguir adelante y a ser capaz de lograr mucho más en la vida.

Al Dr. Carlos Bocanegra García, gracias a sus sabios consejos y su tutoría constante en la realización de este y otros trabajos de investigación.

\section{Referencias}

[18] FERNANDEZ, A. (1987) La inteligencia atrapada. Nueva Visión: Buenos Aires.

[2] MILJANOVICH COSTILLA, M. (2000). "Relaciones entre la inteligencia general, el rendimiento académico y la comprensión de lectura en el campo educativo". Tesis para optar el Grado de Doctor en Educación. Universidad Nacional Mayor de San Marcos, Lima, Perú. Pagina disponible en: http://sisbib.unmsm.edu.pe/Bibvirtual/Tesis/S alud/Reyes_T_Y/contenido.htm.

[3] KERLINGER F. (1988). "Investigación del comportamiento. Técnicas y métodos". Editorial interamericana: México. Pp. 43.

[4] EL TAWAB, S. M. (1997). "Enciclopedia de pedagogía/psicología". Ediciones Trébol: Barcelona. Pp. 183.

[5] http://www.universia.es/portada/actualidad/not icia actualidad.jsp?noticia $=60213$

[6] http://sisbib.unmsm.edu.pe/bibvirtual/Tesis/Sa lud/Reyes_T_Y/Introducci\%C3\%B3n.htm.

[7] http://sisbib.unmsm.edu.pe/BVRevistas/Invest igacion_Psicologia/v10_n1/pdf/a04.pdf

[8] HERNANDEZ SAMPIERI R, FERNADEZ COLLAR C (1998). Metodología de la Investigación Científica, 29Ed. MG, México.

[9] PATTON, B. "A (1958). Study of drops-outs from the Junior Division of the Louisiana State". Dissertation. Abstracts, $\mathrm{N}^{\circ}$ 19: USA. 1958.

[10] LATIESA, M. (1986) Estudio longitudinal de una cohorte de alumnos de la Univ. Autónoma Madrid. En Jornadas sobre demanda de educación superior y rendimiento académico en la Univ. CIDE: Madrid. 1986.

[11] GARCIA VALCARCEL, A.., BLANCO, L. \& ZUBIETA IRUN, J. (1990). Elementos para un análisis evaluativo de la Universidad. El caso de la Universidad de Cantabria. En La investigación educativa sobre la Universidad, actas de las jornadas organizadas por el CIDE y el ICE de la Universidad Politécnica de Madrid. Servicio de Publicaciones del Ministerio de Educación y Ciencia: Madrid. 1990.

[12] GARCIA ARETIO, L. El perfil personal y sociolaboral y su incidencia en el rendimiento académico de los alumnos de la U.N.E.D. En La investigación educativa sobre la Universidad, actas de las jornadas organizadas por el CIDE y el ICE de la Universidad Politécnica de Madrid. Ministerio de Educación y Ciencia: Madrid. 1990.

[13] GARCIA LLAMAS, J. El estudio empírico sobre el rendimiento académico en la enseñanza a distancia. UNED: Madrid. 1986.

[14] McDONALD, J. \& McPHERSON, M. (1975) High School type, sex and socioeconomic factors as predictors of the academic achievement of University students. Educational and Psychological Measurement: USA. 1975.

[15] Universidad Nacional Federico Villarreal, Facultad de Psicología La orientación vocacional y la personalidad. Trabajo monográfico para optar el título de Licenciado en Psicología. Lima ,Perú, 1994.

[16] Meryta Béryl Gutiérrez Briones (1997). Dos casos clínicos incompletos: Trastornos de la conducta por déficit de atención y orientación vocacional. Tesis para optar el título de Licenciado en Psicología. Universidad Nacional Federico Villarreal, Facultad de Psicología. Lima, Perú.

[17] http:/www.nichcy.org/pubs/spanish/fs1stxt.htm

[18] http://www.sccalp.org/padres/aprendizaje.htm

[19] http://www.medisur.sld.cu/index.php/medisur/ announcement/view/5764.

[20] UNIVERSIDAD CATÓLICA DE CHILE (2005), "Seguro salud definiciones". Santiago, Chile. Articulo disponible en: http://www.segurosaluduc.cl/DEFINICIONES. $\mathrm{htm}$

[21] http://es.wikipedia.org/wiki/Bronquitis

[22] http://www.nlm.nih.gov/medlineplus/spanish/e ncy/article/001087.htm

[23] Relación entre las enfermedades más frecuentes con el rendimiento académico de los estudiantes ingresantes de la Universidad Nacional de Trujillo, 2004

E-mail: sandritaa_0202@hotmail.com 